\title{
MODALIDADES DE TRATAMENTO DA ESPASTICIDADE: UMA REVISÃO DA LITERATURA
}

\author{
SPASTICITY TREATMENT MODALITIES: A LITERATURE REVIEW
}

\section{Daniela Hess \\ Julyanna Nishiwaki \\ Mariela Fioriti Liberatori}

Especialização em Fisioterapia Neurofuncional. Faculdade Inspirar. ARCD/AACD - Associação de Reabilitação da Criança Deficiente, Joinville-SC

\author{
Fernando Luis Fischer Eichinger \\ Curso de Fisioterapia \\ Faculdade Guilherme Guimbala \\ Antonio Vinicius Soares \\ Curso de Fisioterapia \\ Faculdade Guilherme Guimbala \\ Universidade da Região de Joinvillear
}

\author{
Contato \\ Antonio Vinicius Soares \\ Rua São José, 490 - Centro - Joinville/SC \\ CEP: 89202-010 \\ E-mail: provinicius.soatrres@gmail.com
}

\section{RESUMO}

Introdução: A espasticidade é uma alteração do tônus muscular bastante comum na prática clínica que, associada a outros sintomas, resulta em limitações importantes para os pacientes. Existe uma grande variedade de métodos para tratamento desse sintoma, contudo, ainda há uma escassez de estudos voltados para identificação de quais as melhores e mais efetivas modalidades de tratamento. Objetivo: Expor e discutir as diversas modalidades de tratamento para a espasticidade disponíveis na atualidade, apontando os melhores resultados com a aplicação desses recursos terapêuticos na prática clínica. Métodos: Realizou-se uma busca nas bases de dados SciELO, LILACS, MEDLINE e Periódicos CAPES. O período de coleta dos dados foi entre 2007 e 2017, por meio dos descritores espasticidade (spasticity) e tratamento (treatment). Resultados: Foram encontradas as seguintes modalidades de tratamento: farmacológico (baclofeno, benzodiazepínicos, dantrolene sódico, gabapentina, tizanidina, toxina botulínica tipo A e fenol); fisioterapêutico (eletroestimulação, cinesioterapia, crioterapia, termoterapia, fisioterapia aquática, terapia vibratória e equoterapia); cirúrgico (rizotomia, tenotomia e transposição tendinosa); e órteses. Conclusões: Destaca-se o uso da toxina botulínica tipo A, apresentando bons resultados e poucos efeitos colaterais, porém, o alto custo e a curta duração dos efeitos são limitações importantes. Merece destaque, ainda, a cinesioterapia mediante técnicas de alongamento como uma importante opção de tratamento da espasticidade, com efeitos imediatos da aplicação e por se tratar de um procedimento simples e fácil; muito embora, ainda não estejam claras as recomendações quanto ao número de repetições, amplitude de movimento realizado, tipo de alongamento e tempo do efeito terapêutico da técnica.

Palavras-chave: Terapêutica. Espasticidade muscular. Hipertonia Muscular. 


\section{ABSTRACT}

Introduction: Spasticity is an alteration in muscle tone, which is quite common in clinical practice and, together with other symptoms, results in important limitations for patients. There is a wide variety of methods for treating this symptom, however, there is still a shortage of studies aimed at identifying which are the best and most effective treatment modalities. Objective: To expose and discuss the various modalities of treatment for spasticity available currently, pointing out the best results with the application of these therapeutic resources in clinical practice. Methods: A research was carried out in SciELO, LILACS, MEDLINE and CAPES Periodical databases. The period of data collection was between 2007 and 2017, through the descriptors spasticity and treatment. Results: The following treatment modalities were found: pharma- cological (baclofen, benzodiazepines, dantrolene sodium, gabapentin, tizanidine, botulinum toxin type $A$ and phenol); physiotherapeutic (electrical stimulation, kinesiotherapy, cryotherapy, thermotherapy, hydrotherapy, vibratory therapy and equine therapy); surgical (rhizotomy, tenotomy and tendon transposition); and orthoses. Conclusions: The use of botulinum toxin type A stands out, with good results and few side effects, but the high cost and short duration of effects are important limitations. Kinesiotherapy through stretching techniques are an important option for the treatment of spasticity, with immediate effects of the application and because it is a simple and easy procedure. Although the recommendations regarding the number of repetitions, range of motion performed, techniques and therapeutic effect time are not yet clear.

Keywords: Therapeutics. Muscle spasticity. Muscle hypertonia.

\section{INTRODUÇÃO}

A espasticidade é uma manifestação comum encontrada na prática clínica em pacientes adultos e pediátricos que gera limitações nas suas tarefas motoras básicas, como no autocuidado, independência e mobilidade ${ }^{1}$. Essa disfunção neurológica no tônus muscular decorre de lesões causadas no neurônio motor superior, ou seja, lesões que acometem o sistema nervoso central (SNC), envolvendo uma grande variedade de patologias agudas e crônicas, tais como o Acidente Vascular Encefálico (AVE)2, Traumatismo Cranioencefálico (TCE), Esclerose Múltipla, Lesões Medulares, e Paralisia Cerebral (PC) 1 .

Não há um consenso na literatura sobre a definição e fisiopatologia da espasticidade. Contudo, em 1980, Lance ${ }^{3}$ foi o primeiro a definir o fenômeno como sendo "uma desordem motora caracterizada por um aumento dependente da velocidade dos reflexos de estiramento tônico (tônus muscular) com movimentos bruscos do tendão" e que resulta da "hiperexcitabilidade do reflexo de estiramento, como um componente do neurônio motor superior". Clinicamente, pode manifestar-se por hipertonicidade, reflexos osteotendinosos aumentados, espasmos musculares e, por vezes, clônus. Está associada a vários sinais e sintomas, entre eles, a fraqueza muscular e a redução da amplitude de movimento ${ }^{4}$. Já para McClelland et al. ${ }^{5}$, a espasticidade resulta da excessiva excitação motoneuronal que leva à contração muscular involuntária em resposta à velocidade aumentada do movimento (velocidade dependente), a qual atualmente é incurável.

Há uma grande variedade de tipos de abordagem para tratamento da espasticidade, entre elas, modalidades fisioterapêuticas (cinesioterapia, eletroterapia, fisioterapia aquática, crioterapia, terapia vibratória), farmacológicas como medicamentos orais, injetáveis ou intratecais, cirúrgicas (rizotomia posterior seletiva, tenotomias); e uso de órteses ${ }^{1}$.

Embora a relevância clínica da espasticidade seja conhecida pela grande incidência e impacto na funcionalidade dos pacientes, ainda existe a necessidade de identificar as melhores e mais efetivas modalidades de tratamento e, assim, estabelecer claras diretrizes de tratamento para essa incapacitante disfunção neurológica. Portanto, o objetivo desta revisão foi levantar e discutir as diversas modalidades de tratamento disponíveis na atualidade sobre a espasticidade e, dessa forma, apontar os melhores resultados com a aplicação desses recursos terapêuticos na prática clínica. 


\section{MÉTODO}

E ste estudo foi delineado como uma pesquisa bibliográfica, realizada por meio de uma revisão de literatura. Para tanto, foram realizadas buscas nas bases de dados SciELO, LILACS, MEDLINE e Periódicos CAPES, com período compreendido entre 2007 e 2017 (exceto a referência histórica clássica de Lance3). Foram utilizados os descritores: espasticidade e tratamento, com seus correspondentes na língua inglesa (spasticity e treatment). Inicialmente foram recrutados 214 artigos escritos em português e inglês, que apresentavam os descritores no título. Após, foram mantidos apenas os artigos originais e de revisão. Por fim, foi realizada a leitura dos resumos para identificação dos artigos que seriam utilizados como resultados do estudo, correspondendo aos trabalhos que apresentavam conteúdo potencialmente relevante. $O$ número total final de artigos elegíveis foi de 60 , sendo 23 originais e 37 de revisão (Figura 1).

\section{RESULTADOS E DISCUSSÃO}

\section{Espasticidade: dados epidemiológicos}

O distúrbio mais frequente das desordens do neurônio motor superior é a espasticidade, sendo que o abalo em alguma estrutura desse meio pode comprometer seriamente o movimento de alguns indivíduos. Por característica, esses distúrbios são observados nos casos de AVE², TCE, Esclerose Múltipla, Lesões Medulares e em comprometimentos congênitos como a PC ${ }^{1}$. Especificamente nos casos de AVE, essa manifestação é muito comum, apresentando incidência em aproximadamente $90 \%$ dos casos na fase crônica da doença ${ }^{6}$.

\section{Avaliação da espasticidade}

$\mathrm{Na}$ avaliação da espasticidade, são utilizados indicadores qualitativos e quantitativos, por meio dos quais é possível identificar a intensidade do tônus muscular e a influência no desempenho da função ${ }^{7}$. Esses instrumentos de avaliação são fundamentais para o norteamento da melhor escolha para o tratamento.

A Escala de Ashworth Modificada (Modified Ashworth Scale) é amplamente utilizada na prática clínica, pois, por meio da mobilização passiva em flexão e extensão dos membros, pode-se avaliar o tônus muscular. Trata-se de uma escala que consiste em 6 pontos para graduar a resistência muscular ante a mobilidade passiva do segmento ${ }^{8}$.

A Escala de Tardieu gradua a rigidez elástica
Figura 1 - Fluxograma detalhando os procedimentos de identificação, seleção, elegibilidade e inclusão dos estudos

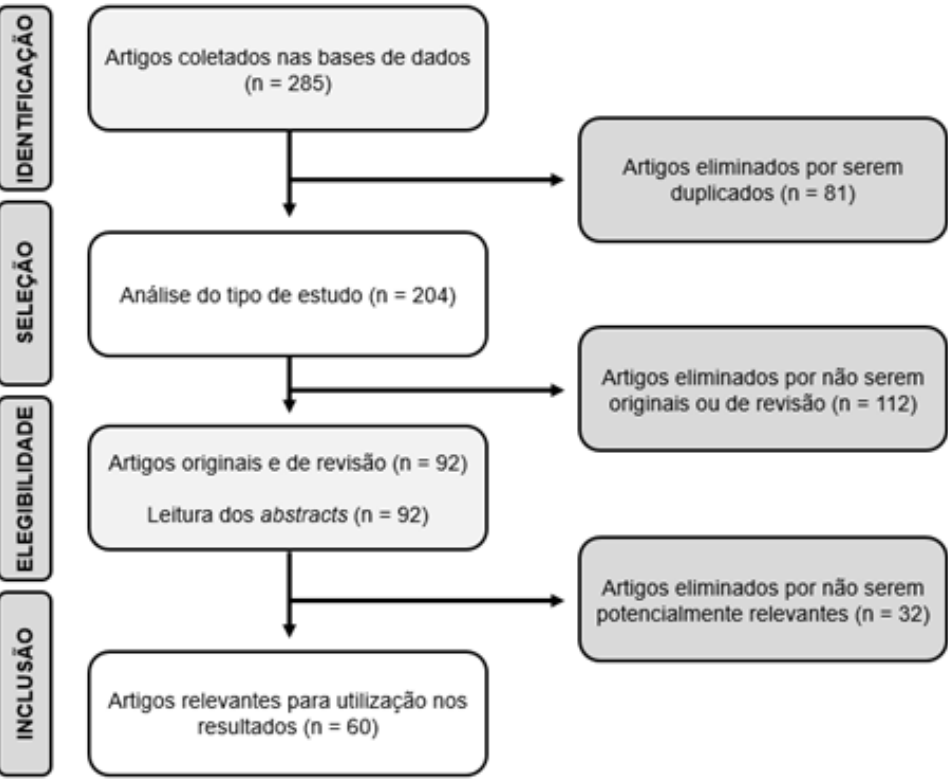

conforme o ângulo de maior resistência e presença de clônus, graduando quantitativamente a gravidade da espasticidade, sendo uma escala ordinal que varia de 0 a $4^{9}$. Outra escala menos utilizada é a Escala de Penn, que consiste em um escore de frequência de espasmos ${ }^{10}$.

A medida do Limiar do Reflexo do Estiramento Tônico, também denominado Limiar do Reflexo do Estiramento Dinâmico, é representada pelo ângulo articular em que os motoneurônios e os respectivos músculos envolvidos em determinada articulação estão sendo recrutados, sendo dependente da velocidade de estiramento. Portanto, quanto mais rápido o estiramento, mais precocemente a contração muscular acontece ${ }^{11}$. 


\section{Modalidades de tratamento}

O reconhecimento das manifestações clínicas do paciente espástico e a quantificação de sua magnitude constituem uma etapa fundamental na correta programação do tratamento a ser instituído ${ }^{7}$.

Diversos recursos terapêuticos podem ser utilizados para o tratamento da espasticidade, tais como: recursos físicos, químicos e o tratamento cirúrgico. A seguir, serão apresentadas as principais modalidades de tratamento.

\section{Farmacológicas}

Os fármacos mais utilizados atualmente são: baclofeno, benzodiazepínicos, dantrolene sódico, gabapentina e cloridrato de tizanidina.

\subsection{Baclofeno}

O baclofeno é uma droga inibitória pré-sináptica que causa ativação dos receptores do ácido gama-aminobutírico (GABA), diminuindo a liberação de neurotransmissores excitatórios, inibindo assim os reflexos mono e polissinápticos, exercendo sua função antiespástica. No entanto, esse medicamento atravessa com dificuldade a barreira hematoencefálica (BHE), sendo necessário recorrer a elevadas doses por via oral, para a obtenção de concentrações eficazes a nível do líquor ${ }^{12}$.

A administração do baclofeno pode ser por via intratecal ou oral. Quando administrado por via oral, é rapidamente absorvido no trato gastrointestinal, possuindo uma semivida de aproximadamente três horas e meia. Uma vez que é parcialmente metabolizado no fígado (15\%) e excretado pelo rim, deve ser utilizado com precaução em doentes com insuficiência hepática ou renal. Seus efeitos colaterais são consideráveis, tais como: sedação, problemas respiratórios, e em doses mais elevadas, fraqueza muscular. Além disso, muitas vezes o efeito sobre a espasticidade é insuficiente ${ }^{13}$.

O tratamento farmacológico com o baclofeno ainda é questionável, haja vista que a interrupção da administração oral pode acarretar diversos problemas, tais como: aumento do tônus muscular e da frequência de espasmos musculares, convulsões prolongadas, hipertermia, disestesia, alucinações ou mesmo falência múltiplas de órgãos ${ }^{14}$.

\subsection{Benzodiazepínicos}

Este fármaco aumenta a afinidade do GABA para o receptor GABA-A, ou seja, aumenta a inibição pré-sináptica que, por consequência, diminui a via de reflexo espinhal. Dessa forma, é desencadeada uma redução da hipercontração muscular, podendo ocasionar efeitos adversos mais severos, como a fraqueza muscular e a sonolência ${ }^{15}$.

\subsection{Dantrolene sódico}

Consiste em um dos primeiros medicamentos utilizados via oral, atuando como um relaxante muscular que inibe a liberação de cálcio do retículo endoplasmático para o citoplasma, etapa fundamental no mecanismo de contração-excitação do músculo esquelético ${ }^{2,16}$. Um dos efeitos colaterais citados é a hepatotoxicidade, portanto a função hepática deve ser constantemente monitorada ${ }^{17}$.

\subsection{Gabapentina}

Este medicamento é um anticonvulsivante que apresenta estrutura semelhante ao GABA, mas que não possui afinidade aos receptores $\mathrm{GABA}^{18}$. É normalmente utilizado para o tratamento da dor neuropática e, em doses elevadas, mostrou eficácia na redução da espasticidade ${ }^{2}$. Seu mecanismo de ação não é bem compreendido, porém, sabe-se que pode apresentar efeitos adversos, como desmaios, sonolência, ataxia, dor de cabeça e tremores ${ }^{15}$.

\subsection{Cloridrato de tizanidina}

Outra opção para o tratamento oral é o cloridrato de tizanidina (Tizanidina). Este é um fármaco utilizado com menor frequência, por se tratar de uma novidade e por trazer efeitos secundários, como sedação, hipotensão, astenia, tonturas, alucinações ou hepatotoxicidade ${ }^{13,19}$. É uma droga com ação central, antagonista dos receptores adrenérgicos, que aumenta a inibição sináptica dos neurônios motores.

\subsection{Bloqueadores químicos}

No tratamento medicamentoso da espasticidade, os bloqueadores químicos mais utilizados são: toxina botulínica tipo A, fenol e a bomba de baclofeno. Essas medicações atuam por diferentes mecanismos que resultam em diminuição da excitabilidade dos reflexos espinhais ${ }^{2,20}$.

\subsubsection{Toxina botulínica tipo $A$}

A toxina botulínica tipo A (TBA) tem se mostrado muito eficaz no tratamento da espasticidade, sendo considerada uma opção terapêutica para tratamento de doenças neurológicas causadoras da hiperatividade muscular ${ }^{21,22}$. O mecanismo de ação da toxina consiste na inibição da liberação de acetilcolina na junção neuromuscular, resultando em uma diminuição da contração muscular.

De acordo com Silva Junior, Dimbany e Tôrres $^{23}$, "sua ação se dá por duas vias, afetando as fibras musculares esqueléticas extrafusais e intrafusais, influenciando o feedback aferente e o influxo ao motoneurônio alfa". Sua ação não interfere na produção ou armazenamento da acetilcolina, pois, à medida que o efeito neurotóxico começa a decair, 
as junções neuromusculares restauram-se e os brotamentos axonais involuem; como consequência, a espasticidade retorna. Portanto, a ação farmacológica vigora por tempo determinado, tendo uma variação, de acordo com a literatura, entre 3 e 12 meses. Graham $^{24}$ descreve em seu estudo que os efeitos secundários são temporários, assim como os efeitos de tratamento, que duram de 3 a 4 meses. Dessa forma, é necessária a aplicação periódica desse fármaco para o controle da espasticidade ${ }^{25,26}$.

O encaminhamento para a aplicação deve ser direcionado para cada condição clínica, sendo o médico e o fisioterapeuta os profissionais responsáveis pela indicação. Para melhor localização do ponto motor, utilizam-se técnicas de eletroestimulação, em que é feita a localização precisa do músculo que necessita ser tratado ${ }^{27}$. A seleção dos músculos principais para aplicação, em pacientes com paralisia cerebral, por exemplo, leva em consideração músculos em risco imediato de desenvolver contraturas, impedindo o próximo estágio do desenvolvimento motor ${ }^{2,28}$.

De acordo com Chinelato, Perpétuo e Krueger Beck $^{29}$, embora a TBA demonstre-se como uma técnica eficiente para a redução do tônus muscular, seu efeito se dá pela paralisação temporária da musculatura afetada, e não pela recuperação da função muscular próxima dos níveis normais. Por esse motivo, sua aplicabilidade limita-se, até o presente momento, aos efeitos locais e temporários obtidos no aumento da excursão dos tecidos moles adjacentes ao tecido da aplicação.

\subsubsection{Fenol}

O fenol é um agente neurolítico usado para o bloqueio de nervos que possuem pequena função sensorial30. Esse tipo de bloqueio tem sido usado em pontos motores musculares em casos de espasticidade focal ou multifocal.

Ponto motor é a área no músculo onde estão localizadas as maiores concentrações de junções mioneurais ${ }^{31}$. A concentração recomendada é de $5-7 \%$ de Fenol e $45-100 \%$ de Álcool. A administração do Fenol implica alguns resultados indesejáveis, como dor, disestesias devido à necrose de axônios sensoriais nos nervos periféricos, letargias e náuseas secundárias à absorção sistêmica e uma potencial necrose na região da aplicação ${ }^{15,31,32}$.

De acordo com Trevisol Bittencourt e Tournier $^{32}$, o mecanismo de ação do Fenol é composto por duas fases: na primeira, "o fármaco atua como anestésico sobre as fibras Gamas causando inativação dos fusos musculares e inibição dos reflexos de estiramento", já na segunda, em seguida, "ocorre a desnaturação proteica (proteolize) com interrupção dos sinais eferentes das células hiperexcitáveis do corno anterior da medula, levando a uma necrose axonal induzida chamada de degeneração valeriana". Cabe destacar que os tubos endoneurais permanecem íntegros.

\subsubsection{Bomba de baclofeno}

O baclofeno intratecal é mais eficaz por agir diretamente no líquido cefaloraquidiano (LCR), por meio de uma bomba programável, dando concentrações mais elevadas nos tecidos relevantes e melhor resultado terapêutico com menos efeitos colaterais ${ }^{13}$. Geralmente está indicado para pacientes com espasticidade grave, com finalidade de reduzir ou prevenir as contraturas musculares, aumentar o conforto, meIhorar o posicionamento e facilitar os cuidados em pacientes com incapacidade funcional grave, quando outras formas de tratamento falharam ${ }^{33}$.

\section{Fisioterapêuticas}

\subsection{Eletroestimulação}

A Estimulação Elétrica Funcional (FES) é uma corrente que tem como base a produção de contração muscular por meio de estimulação elétrica, que despolariza o nervo motor, produzindo uma resposta sincronizada em todas as unidades motoras, promovendo assim uma contração eficiente ${ }^{34}$.

De acordo com Schuster, Sant e Dalbosco ${ }^{35}$, a FES é empregada no controle da espasticidade devido a mecanismos imediatos e tardios, demonstrados na literatura. Os efeitos imediatos são: inibição recíproca, relaxamento do músculo espástico e estimulação sensorial de vias aferentes. Já os efeitos tardios agem na neuroplasticidade e são suscetíveis para modificar as propriedades viscoelásticas musculares, além de favorecerem a ação e o desenvolvimento de unidades motoras de contração rápida.

Sposito $^{36}$ descreve em seu estudo que a eletroestimulação é utilizada para redução da hipertonicidade grave, sobre os músculos antagonistas, em que a estimulação dos padrões aferentes resulta na inibição dos músculos agonistas, com redução do seu tônus. O uso contínuo oferece um decréscimo nas contraturas, aumento na atividade motora dos músculos agonistas além de reduzir o tônus de músculos antagonistas.

A estimulação elétrica neuromuscular (NMES) é a ação da estimulação elétrica sobre o tecido muscular por meio do sistema nervoso periférico (SNP) intacto. Existem seis categorias principais de programas de tratamento que utilizam a NMES, agrupadas de acordo com os objetivos do tratamento, sendo elas: aumentar a força ou manter a massa muscular durante e após períodos de inatividade forçada; manter ou ganhar amplitude de movimento; reeducar 
ou facilitar o controle motor voluntário; reduzir temporariamente os efeitos da espasticidade; fornecer suporte ortésico e reduzir a formação de edema ${ }^{37}$.

Alguns pacientes podem apresentar certo grau de intolerância sensorial aos programas de NMES, normalmente esse fator não é indicativo de problemas no SNP, mas indica que uma parte da informação sensorial artificial excessiva está sendo interpretada pelo SNC como desconfortável. As aplicações terapêuticas da NMES são sempre realizadas por meio de eletrodos de superfície, por esse motivo, os nervos sensoriais da pele sempre receberão a maior concentração de corrente elétrica, e alguns nervos sempre serão invariavelmente ativados antes que exista a excitação do neurônio motor ${ }^{38}$.

Diante da complexidade das patologias, a efetividade dos tratamentos com a NMES varia de acordo com quais estruturas do SNC são mais sensíveis a características particulares da NMES e a fonte da espasticidade. Essa técnica que promove contração muscular por meio de uma corrente elétrica aplicada tem promovido efeitos benéficos em pacientes neurológicos, como, por exemplo: diminuição da atrofia muscular e da espasticidade, aumento da força muscular e facilitação da recuperação do movimento ${ }^{39}$.

O efeito neurofisiológico proposto para cada tipo de tratamento é mediado por meio da inibição sináptica do motoneurônio, portanto as frequências de estimulação podem ser ajustadas de modo relativamente alto, aumentando a quantidade de informação para o SNC. Os programas para o controle da espasticidade parecem ter melhores resultados, quando seguidos imediatamente de outras formas de terapia, tais como facilitação neuromuscular proprioceptiva (FNP), ganho de força e treino funcional ${ }^{40}$.

Uma recente revisão sistemática realizada por Stein et al. ${ }^{41}$ sobre o uso da NMES para pacientes hemiparéticos por AVC mostrou que esse recurso quando combinado com outras técnicas de tratamento, como a cinesioterapia e/ou a TBA, pode ser uma boa opção de tratamento, proporcionando redução da espasticidade e aumento da amplitude de movimento. Esses efeitos terapêuticos da NMES podem ser explicados pelo aumento de ativação das fibras lb, mecanismo de inibição recorrente via célula de Renshaw, no aumento dos estímulos sensoriais cutâneos e na inibição recíproca via estimulação dos músculos antagonistas dos grupos musculares espástico ${ }^{41}$.

\subsection{Cinesioterapia}

O alongamento tem como objetivo normalizar o tônus muscular para manter ou aumentar a extensibilidade dos tecidos moles, reduzindo a dor e meIhorando a função. Em pacientes com espasticidade, normalmente o tratamento com a utilização do alon- gamento é de longo prazo, realizado diariamente ou semanalmente ${ }^{42}$.

Bovend'Eerdt et al. ${ }^{43}$ realizaram uma revisão sistemática sobre os efeitos do alongamento na espasticidade, concluíram que embora haja evidência positiva apoiando os efeitos imediatos do alongamento após uma sessão, não se sabe por quanto tempo eles permanecem e suas consequências a longo prazo. Portanto, os resultados ainda são inconclusivos ao uso das técnicas de alongamento.

O método de FNP é baseado principalmente nos mecanismos neurofisiológicos que envolvem o reflexo de estiramento. Essa técnica utiliza a facilitação que resulta em um aumento da excitabilidade, auxiliando nos músculos enfraquecidos, sendo que a inibição é a diminuição da excitabilidade, em que a espasticidade do músculo seria diminuída ${ }^{44}$.

O Conceito Bobath é baseado na neuroplasticidade, utilizando da facilitação, normalização e adequação de tônus, reeducação do movimento por meio dos manuseios, em pontos-chave específicos, que auxiliam no desenvolvimento do movimento desejado. Esse método pode ser utilizado para diversas finalidades, entre elas, verificar o comportamento neuromuscular em algumas patologias ou lesões que afetem sistema nervoso, bem como o efeito da falta de musculatura no nível da ativação muscular ${ }^{45}$.

Os manuseios aplicados por meio do Bobath influenciam o tônus muscular por meio da mobilização, alongamento e ativação de músculos, partindo do princípio da manipulação de pontos-chave, o que facilita o controle postural e desempenho das atividades de vida diárias (AVDs) ${ }^{2}$. As manipulações propostas por esse conceito promovem respostas motoras automáticas, resultando em um controle funcional mais efetivo. Atuam ainda sobre o componente sensório-motor do tônus muscular, reflexos, padrões de movimentos anormais, controle postural, sensação, percepção e memória cinestésica ${ }^{45,46}$.

$\mathrm{Na}$ questão da característica neural, os $\mathrm{Pa}$ drões Influenciando Tônus (PITs), alteram reflexos cutâneos e proprioceptivos, inicialmente recrutando o sistema de feedback e, em longo prazo, o sistema de feedforward. Os PITs são aplicados com intuito de produzir modificações no corpo inteiro (uso de pontos-chave mais proximais) ou mobilizações específicas bem localizadas.

Peres, Ruedell e Diamante ${ }^{47}$ concluíram em seu estudo que é durante o desempenho de AVDs que a incapacidade da criança é manifestada, em que a limitação funcional é percebida. Todas as argumentações feitas na teoria sobre a influência do tônus e da força muscular, e sua correlação com as atividades funcionais, foram demonstradas neste estudo, podendo-se afirmar que houve uma diminuição 
do tônus e aumento da força muscular com consequente melhora das atividades funcionais estáticas.

\subsection{Crioterapia e termoterapia}

A crioterapia para o tratamento da espasticidade, em uso prolongado, tem como objetivo reduzir a atividade do fuso muscular por aumentar seu limiar de disparo. A neurotransmissão de impulsos eferentes e aferentes é diminuída, assim ocorre a redução da velocidade de condução nervosa e da transmissão neuromuscular, inibindo a espasticidade. Sua aplicação, para a redução dessa manifestação, apresenta uma variação de temperatura de $0^{\circ}$ a $18,3^{\circ} \mathrm{C}$, em um tempo estimado de 25 a 30 minutos para diminuir a temperatura muscular ${ }^{7}$. O efeito do frio permanece, após sua aplicação, por cerca de 30 minutos a 2 horas, servindo como um meio facilitador da cinesioterapia. Sua aplicação pode ser administrada de três formas diferentes: a imersão em água fria, bolsas de gelo ou deslizamento de cubos de gelo sobre a pele e Sprays de evaporação, como o cloreto de etila ${ }^{7,48}$.

A aplicação local de calor produz um efeito analgésico e de relaxamento muscular, por diminuir o limiar de disparo dos eferentes gama, reduzir a excitabilidade dos fusos musculares e aumentar a atividade dos órgãos tendinosos de Golgi. Portanto, o frio e o calor são utilizados como recursos auxiliadores, facilitando a realização das técnicas da cinesioterapia. Estudos relatam a eficácia na utilização da crioterapia e termoterapia para a redução da espasticidade, quando estas são combinadas com outras terapias, por serem vantajosas pelos efeitos fisiológicos, apresentando baixo custo e pela possibilidade de uso domiciliar?

\subsection{Fisioterapia aquática}

Trata-se de um recurso que se utiliza dos efeitos físicos e fisiológicos do meio aquático aquecido, por meio da imersão, como auxiliador da reabilitação. Possui como efeito terapêutico o alívio das dores e do espasmo muscular, relaxamento, manutenção ou aumento da amplitude de movimento das articulações, redução da espasticidade muscular, fortalecimento dos músculos e melhora das atividades funcionais ${ }^{49}$.

Para utilização desse recurso, o terapeuta deve compreender os efeitos físicos e fisiológicos que a água oferece, assim como suas propriedades, favorecendo a proposta de tratamento ${ }^{50}$. As propriedades da água que influenciam na redução do tônus são: o empuxo, que diminui o peso exercido pela ação da gravidade; e a flutuabilidade, que, quando bem utilizada, auxilia o movimento, tornando-o mais fácil. Ambas diminuem o recrutamento de tônus. Outra explicação para a diminuição de tônus seria o fato de que, durante a imersão, os impulsos aferentes táteis e proprioceptivos são reduzidos, ocorrendo uma diminuição da emissão de estímulos facilitadores aos músculos extensores e dessensibilização do fuso muscular, reduzindo momentaneamente a atividade das fibras gama ${ }^{51}$. Com essas informações aferentes reduzidas, ocorre uma reação mais organizada ao estímulo gravitacional, fazendo com que pacientes espásticos controlem seus movimentos de maneira mais normal ${ }^{50,51}$.

A temperatura da água é mais um fator que diminui o tônus muscular, quando esta é aquecida na faixa de $32^{\circ}$ a $33^{\circ} \mathrm{C}$, pois o calor inibe atividade tônica. De acordo com Orsini et al..$^{51}$, "existe certo consenso sobre os benefícios do ambiente aquático para o tratamento de pacientes com lesões neurológicas que cursam com espasticidade, porém há divergências quanto á abordagem terapêutica específica a ser utilizada em seu embasamento científico".

Métodos como BadRagaz, Halliwick e Watsu podem ser utilizados como técnicas no tratamento da espasticidade ${ }^{51}$. Dimitrijević et al. ${ }^{52}$ realizaram um programa de exercícios aquáticos com o objetivo de melhoria no desempenho funcional, tônus muscular e resistência cardiorrespiratória em crianças com paralisia cerebral espástica. Concluíram que os resultados foram positivos para os objetivos propostos, sendo que o grau de espasticidade foi avaliado por meio da escala de Ashworth, apresentando diminuição considerável após o período de intervenção.

\subsection{Terapia vibratória}

As vibrações implicam múltiplas influências fisiológicas, promovendo padrões normais de atividade motora pela modulação da excitabilidade dos motoneurônios. Elas aumentam o influxo aferente final do fuso muscular primário, permitindo a contração reflexa, o chamado reflexo tônico de vibração. Essa terapia provoca efeitos inibitórios em padrões espásticos e, por conseguinte, efeitos no controle motor ${ }^{53,54}$.

\subsection{Equoterapia}

Consiste em um método terapêutico que utiliza o cavalo como instrumento de trabalho, para auxiliar no desenvolvimento motor, emocional e social de pessoas com deficiência, baseado na prática de atividades equestre e técnicas de equitação. O paciente é submetido ao o movimento do cavalo, este atua diretamente no cérebro e, em seguida, reflete no corpo, pois é direcionado ao ajuste postural ${ }^{55}$.

Dessa forma, a equoterapia contribui para o desenvolvimento do tônus, forma muscular, percepção corporal, coordenação motora, entre outros aspectos. O ajuste do tônus é a primeira manifestação quando o paciente é colocado sobre o cavalo, pois, para todos os movimentos realizados pelo animal, é necessária uma adequação da musculatura como resposta aos desequilíbrios provocados ${ }^{56}$. 
Por exemplo, no movimento de antepistar do cavalo, é produzida uma frequência alta por intermédio do curto comprimento do passo. Os receptores proprioceptivos intrafusais, que respondem com estímulos rápidos, agem nos receptores articulares, assim, como resposta, tem-se um aumento súbito do tônus, tendo indicação para pacientes com quadro de hipotonia. Já durante o movimento de transpistar, o cavalo apresenta um passo longo que proporciona maior número de estímulos aos praticantes, transmitindo uma baixa frequência de passos, diminuindo a velocidade dos estímulos proprioceptivos, mantendo ritmo e cadência, estimulando lentamente o sistema vestibular, que resulta em uma diminuição do tônus, sendo, portanto, indicado para pacientes portadores de hipertonia ${ }^{57}$.

\section{Cirúrgicas}

\subsection{Rizotomia posterior seletiva}

A rizotomia posterior seletiva é um procedimento cirúrgico realizado sob anestesia geral. A cauda equina é exposta por laminotomia osteoplástica lombar, sendo identificadas as raízes dorsais sensitivas de S1 à L2 bilateralmente, confirmadas após estímulos neurais através de uma lesão de aproximadamente $30 \%$ de seu diâmetro em cada raiz nervosa ${ }^{58,59}$.

Essa técnica é indicada para redução da espasticidade em pacientes que possuem comprometimentos clínicos mais brandos, sendo que auxilia na melhora da funcionalidade, por exemplo, com meIhora em aspectos relacionados com a marcha. Em pacientes com maior comprometimento, pode trazer benefícios como melhora no posicionamento, conforto e facilitando os cuidados com higiene e profilaxia de complicações físicas ${ }^{36,58,60}$.

\subsection{Tenotomias e transposição tendinosa}

As tenotomias são indicadas para liberação de músculos gravemente espásticos, esse procedimento envolve alongamento das estruturas musculotendíneas. No alongamento de tendão, ocorre redução da espasticidade pela alteração dos receptores de Golgi e nos fusos musculares ${ }^{61}$. Pode ser realizada em pacientes que não apresentam movimentação voluntária ${ }^{36}$.

A transposição tendinosa é utilizada em músculos que se apresentam parcialmente funcionais, pois, por meio dessa intervenção, podem ser produzidos movimentos úteis. É utilizada também em pacientes com espasticidade, em que é feita a transferência de hiperatividade isolada de um músculo espástico para onde ele poderá atuar como um antagonista de outro músculo espástico, fazendo com que ocorra um equilíbrio na articulação apesar da presença de espasticidade ${ }^{61}$.

\section{4 Órteses}

São dispositivos estáticos ou dinâmicos aplicados externamente aos membros, sendo utilizados para estabilização, imobilização, prevenção ou correção de deformidades, proteção contra lesões, estimulação e maximização das funções, além da redução do quadro de espasticidade. As órteses, também denominadas "talas", são geralmente utilizadas como complemento do tratamento com outras terapias ${ }^{2,36}$.

As órteses estáticas são frequentemente efetivas na manutenção do comprimento da musculatura envolvida. Quando o músculo é passivamente alongado, acontece uma diminuição da sensibilização do reflexo de estiramento, com redução da espasticidade, podendo permanecer por até três horas após sua remoção ${ }^{36,62}$.

Basaran et al. ${ }^{63}$ realizaram um estudo com o intuito de evidenciar a eficácia da utilização de órtese nos músculos flexores do punho em pacientes pós-AVC, contudo, não obtiveram melhora significativa no quadro de espasticidade. Portanto, ainda existe uma escassez de evidências científicas que comprovem a eficácia do uso desse tipo de recurso. 


\section{CONCLUSÕES}

$\mathrm{N}$ o manejo da espasticidade, é fundamental uma cuidadosa avaliação do paciente, detalhando os grupos musculares envolvidos e mensurando o grau de espasticidade manifestado. Tal procedimento possibilita ao terapeuta elaborar as mais adequadas estratégias de tratamento para esse distúrbio, que habitualmente gera grande impacto negativo sobre a capacidade funcional dos pacientes.

Com relação às modalidades farmacológicas utilizadas, destaca-se o uso da TBA, que tem apresentado respostas favoráveis. Ela apresenta menores efeitos colaterais quando comparada às demais intervenções medicamentosas, porém, como fatores limitantes, existem o alto custo do procedimento e o curto período de duração dos efeitos. Contudo, cabe salientar que esse procedimento pode ser realizado de forma gratuita por intermédio do Sistema Único de Saúde (SUS), tornando-se mais acessível para a população. Em pacientes que apresentam quadro de espasticidade grave, o uso do baclofeno (via oral) também tem apresentado efetividade, além de poucos efeitos colaterais.
Dentro das modalidades fisioterapêuticas, a cinesioterapia, por meio de técnicas de alongamento muscular, é tida como um importante recurso de tratamento, apresentando evidências sobre os efeitos imediatos de aplicação e apontada como a técnica com melhor resultado de diminuição da espasticidade em longo prazo. Além disto, trata-se de um procedimento simples e de fácil aplicabilidade, fatores que contribuem para uma maior utilização na prática clínica. As técnicas de cinesioterapia podem ser utilizadas em combinação com a NMES, em que seu efeito pode ser potencializado.

Pode-se concluir que, na atualidade, existe uma ampla variedade de métodos para o tratamento da espasticidade, no entanto, ainda não existe um consenso sobre as modalidades mais eficazes para a reabilitação. Recomenda-se que o manejo da espasticidade envolva não apenas uma modalidade terapêutica isolada, mas, sim, a combinação de técnicas, permitindo, assim, obter ganhos mais significativos no processo de reabilitação. Embora exista extensa literatura sobre diferentes técnicas e recursos com bons resultados no controle da espasticidade, ainda são imprescindíveis novas pesquisas com maior rigor metodológico para estabelecer diretrizes claras sobre as melhores indicações, eficácia e a relação custo-benefício.

\section{Referências}

1. Nair KP, Marsden J. The management of spasticity in adults. BMJ. 2014;349(1):g4737.

2. Thibaut A, Chatelle C, Ziegler E, Bruno MA, Laureys S, Gosseries O. Spasticity after stroke: physiology, assessment and treatment. Brain Inj. 2013;27(10):1093-1105.

3. Lance J. Spasticity: disorders motor control. In: Feldman RG, Young RP, Koella WP. Symposium synopsis. Miami, Florida. Year Book Medical Publishers, Chicago. 1980:485-494.

4. Logan LR. Rehabilitation techniques to maximize spasticity management. Top Stroke Rehabil. 2011;18(3):03-211.

5. McClelland S 3rd, Teng Q, Benson LS, Boulis NM. Motor neuron inhibition-based gene therapy for spasticity. Am J Phys Med Rehabil. 2007;86(5):412-421.

6. Santos DG, Pegoraro ASN, Abrantes CV, Jakaitis F, Gusman S, Bifulco SC. Evaluation of functional mobility of patients with stroke sequela after treatment in hydrotherapy pool using the Timed Up and Go Test. Einstein. 2011;9(2):302-306.

7. Felice TD, Santana LR. Recursos fisioterapêuticos (crioterapia e termoterapia) na espasticidade: revisão de literatura. Rev Neurocienc. 2009;17(1):57-62.

8. Heidari M, Abootalebi C, Hosseini SA. Validity of Modified Ashworth Scale as a measure of wrist spasticity in stroke patients. Iranian Rehabilitation Journal. 2011;9(13):26-30.

9. Ben Shabat E, Palit M, Fini NA, Brooks CT, Winter A, Holland AE. Intra- and interrater reliability of the Modified Tardieu Scale for the assessment of lower limb spasticity in adults with neurologic injuries. Arch Phys Med Rehabil. 2013;94(12):2494-501.

10. Skogberg O, Samuelsson K, Ertzgaard P, Levi R. Changes in body composition after spasticity treatment with intrathecal baclofen. J Rehabil Med. 2017;49(1):36-39.

11. Calota A, Feldman AG, Levin MF. Spasticity measurement based on tonic strech reflex threshol in stroke using a portable device. Clin Neurophysiol, 2008;119(10): 2329-2337.

12. Luo D, Wu G, Ji Y, Zhang Z, He F, Mou X, et al. The comparative study of clinical efficacy and safety of baclofen vs tolperisone in spasticity caused by spinal cord injury. Saudi Pharm J. 2017;25(4):655-659. 
13. Delgado MR, Hirtz D, Aisen M, Ashwal S, Fehlings DL, McLaughlin J, et al. Practice parameter: pharmacologic treatment of spasticity in children and adolescents with cerebral palsy (an evidencebased review): report of the Quality Standards Subcommittee of the American Academy of Neurology and the Practice Committee of the Child Neurology Society. Neurology. 2010;74(4):336-343.

14. Pérez Arredondo A, Cázares Ramírez E, Carrillo Mora P, Martínez Vargas M, Cárdenas Rodríguez N, Coballase Urrutia $E$, et al. Baclofen in the therapeutic of sequele of traumatic brain injury: spasticity. Clin Neuropharmacol. 2016;39(6):311-319.

15. Lapeyre E, Kuks JB, Meijler WJ. Spasticity: revisiting the role and the individual value of several pharmacological treatments. NeuroRehabilitation. 2010;27(2):193-200.

16. Bannister RA. Dantrolene-Induced Inhibition of Skeletal L-Type Ca2+ Current Requires RyR1 Expression. Biomed Res Int. 2013;2013:1-10.

17. Evans SH, Cameron MW, Burton JM. Hypertonia. Curr Probl Pediatr Adolesc Health Care. 2017;47(7):161-166.

18. Campesatto EA, Marques CEBS. Uso de gabapentina no tratamento da dor neuropática e do topiramato na enxaqueca. Rev. Saúde Desenvolv. 2012;1(1):8-19.

19. Olvey EL, Armstrong EP, Grizzle AJ. Contemporary pharmacologic treatments for spasticity of the upper limb after stroke: a systematic review. Clin Ther. 2010;2(14):2282-2303.

20. Bethoux F. Spasticity management after stroke. Phys Med Rehabil Clin N Am. 2015;26(4):625-639.

21. Sposito MMM. Chemical blockage for cerebral palsy spasticity treatment. Acta Fisiatr. 2010;7(2):68-83.

22. Teles MS, Mello EMCL. Toxina botulínica e fisioterapia em crianças com paralisia cerebral espástica: revisão bibliográfica. Fisioter Mov. (Online). 2011;24(1):181-190.

23. Silva Junior JA, Dimbany KMS, Tôrres AKV. Principais fármacos utilizados no tratamento da espasticidade: uma revisão bibliográfica. Cad Estud Pesq. 2013;17(37):23-32.

24. Graham LA. Management of spasticity revisited. Age Ageing. 2013;42(4):435-441.

25. Ozcakir S, Sivrioglu K. Botulinum toxin in poststroke spasticity. Clin Med Res. 2007;5(2):132-138.

26. Simpson DM, Gracies JM, Graham HK, Miyasaki JM, Naumann M, Russman B, et al. Assessment: Botulinum neurotoxin for the treatment of spasticity (an evidence-based review): report of the Therapeutics and Technology Assessment Subcommittee of the American Academy of Neurology. Neurology. 2008;70(19):1691-1698.

27. Coelho TLS, Ribeiro NMS. Percepção do paciente neurológico sobre os resultados da neurólise química com toxina botulínica. Rev Neurocienc. 2011;19(2):221-228.

28. Ward AB. Spasticity treatment with botulinum toxins. J Neural Transm. 2008;115(4):607-616.

29. Chinelato JCA, Perpétuo AMA, Krueger Beck E. Espasticidade - aspectos neurofisiológicos e musculares no tratamento com toxina botulínica do tipo A. Rev Neurocienc. 2010;18(3):395-400.

30. Elovic EP, Esquenazi A, Alter KE, Lin JL, Alfaro A, Kaelin DL. Chemodenervation and nerve blocks in the diagnosis and management of spasticity and muscle overactivity. PM\&R. 2009;1(9):842-851.

31. Potasz C. Bloqueios químicos como auxiliares na reabilitação de crianças com paralisia cerebral - a importância de uma visão multidisciplinar. Rev Neurocienc. 2010;18(2):172-178.

32. Trevisol Bittencourt PC, Tournier MB. Phenol block for spasticity management. Acta Fisiatr. 2008;15(3):189-191.

33. Kopec K. Cerebral palsy: pharmacologic treatment of spasticity. US Pharm. 2008;33(1):22-26.

34. Vafadar AK, Côté JN, Archambault PS. Effectiveness of functional electrical stimulation in improving clinical outcomes in the upper arm following stroke: a systematic review and meta-analysis. Biomed Res Int. 2015. Epub 2015 Jan 22.

35. Schuster RC, Sant CR, Dalbosco V. Efeitos da estimulação elétrica funcional (FES) sobre o padrão de marcha de um paciente hemiparético. Acta Fisiatr 2007;14(2):82-86.

36. Sposito MMM. Surgeries and physical interventions in the treatment of cerebral palsy spasticity. Acta Fisiatr. 2010;17(2):84-91.

37. Sheffler LR, Chae J. Neuromuscular electrical stimulation in neurorehabilitation. Muscle Nerve. 2007;35(5):562-590.

38. Krueger Beck EK, Scheeren EM, Nogueira Neto GN, Button VLSN, Nohama P. Efeitos da estimulação elétrica funcional no controle neuromuscular artificial. Rev Neurocienc. 2011;19(3):530-541.

39. Knutson JS, Fu MJ, Sheffler LR, Chae J. Neuromuscular electrical stimulation for motor restoration in hemiplegia. Phys Med Rehabil Clin N Am. 2015;26(4):729-745. 
40. Hatzis A, Stranjalis G, Megapanos C, Sdrolias PG, Panourias IG, Sakas DE. The current range of neuromodulatory devices and related technologies. Acta Neurochir Suppl. 2007;97(1):21-29.

41. Stein C, Fritsch CG, Robinson C, Sbruzzi G, Plentz RDM. Effects of electrical stimulation in spastic muscles after stroke. Systematic review and meta-analysis of randomized controlled trials. Stroke. 2015;46(8):2197-2205.

42. Nielsen JB, Crone C, Hultborn $\mathrm{H}$. The spinal pathophysiology of spasticity--from a basic science point of view. Acta Physiol (Oxf). 2007;189(2):171-180.

43. Bovend'Eerdt TJ, Newman M, Barker K, Dawes H, Minelli C, Wade DT. The effects of stretching in spasticity: a systematic review. Arch Phys Med Rehabil. 2008;89(7):1395-1406.

44. Wang J, Lee S, Moon S. The immediate effect of PNF pattern on muscle tone and muscle stiffness in chronic stroke patient. J. Phys. Ther. Sci. 2016;28(3):967-970.

45. Graham JV, Brock CEK, Swain E, Irwin Carruthers S. The Bobath concept in contemporary clinical practice. Top Stroke Rehabil. 2009;16(1):57-68.

46. Mikolajewska E. NDT-Bobath method in normalization of muscle tone in post-stroke patients. Adv Clin Exp Med 2012;21(4):513-517.

47. Peres LW, Ruedell AM, Diamante C. Influência do Conceito Neuroevolutivo Bobath no tônus e força muscular e atividade funcionais estáticas e dinâmicas em pacientes diparéticos espásticos após paralisia cerebral. Rev Saúde. 2009;35(1):28-33.

48. EI Maksoud GMA, Sharaf MA, Rezk Allah SS. Efficacy of cold therapy on spasticity and hand function in children with cerebral palsy. J Adv Res. 2011;2(4):319-325.

49. Tonieto $M$, Rama $P$, Schuster RC, Renosto $A$. Efeitos de uma intervenção de fisioterapia aquática em pacientes pós-acidente vascular cerebral. Rev Atenção Saúde. 2015;13(45):5-12.

50. Bonomo LMM, Castro VC, Ferreira DM, Miyamoto ST. Hidroterapia na aquisição da funcionalidade de crianças com Paralisia Cerebral. Rev Neurocienc 2007;15(2):125-130.

51. Orsini M, Feitas MRG, Mello MP, Antonioli RS, Kale N, Eigenheer JF, et al. Hidroterapia no gerenciamento da espasticidade nas paraparesias espásticas de várias etiologias. Rev Neurocienc. 2010;18(1):81-86.

52. Dimitrijević L, Bjelaković B, Lazović M, Stanković I, Čolović $H$, Kocić M, et al. Aquatic exercise in the treatment of children with cerebral palsy. Srp Arh Celok Lek. 2012;140(11-12):746-750.

53. Krueger Beck EK, Neto GNN, Nohama P. Estímulo vibracional na espasticidade - uma perspectiva de tratamento. Rev Neurocienc. 2010;18(4):523-530.

54. Silva JM, Lima MO, Paula Júnior AR. Efeito agudo da estimulação vibratória em hemiparéticos espásticos pós-acidente vascular encefálico. Rev Bras Eng Bioméd. 2011;27(4):224-230.

55. Vermöhlen V, Schiller P, Schickendantz S, Drache M, Hussack S, Gerber Grote A, et al. Hippotherapy for patients with multiple sclerosis: A multicenter randomized controlled trial (MS-HIPPO). Mult Scler. 2017. Epub 2017 Aug 1.

56. Chae-Woo L, Seong GK, Sang SN. The effects of hippotherapy and a horse riding simulator on the balance of children with cerebral palsy. J Phys Ther Sci. 2014;26(3):423-425.

57. Araujo TB, Silva NA, Costa JN, Pereira MM, Safons MP. Efeito da equoterapia no equilíbrio postural em idosos. Rev Bras Fisioter. (Online). 2011;15(5):414-419.

58. Val JAC, Lima ALO, Martins AO. O impacto da rizotomia dorsal seletiva na qualidade de vida de crianças espástica portadoras de paralisia cerebral. Arq Bras Neurocir. 2008;27(1):7-11.

59. Morota N, Ihara S, Ogiwara H. Neurosurgical management of childhood spasticity: functional posterior rhizotomy and intrathecal baclofen infusion therapy. Neurol Med Chir (Tokyo). 2015;55(8):624-639.

60. Jucá RVBM, Jucá CEB, Caldas CAT, Ioriatti ES, Baptista CRJA, Machado HR. O efeito da rizotomia dorsal seletiva no quadro clínico e nos cuidados diários de crianças com paralisia cerebral espástica. Acta Fisiatr. 2011;18(1):11-15.

61. Damiano DL, Alter KE, Chambers $\mathrm{H}$. New clinical and research trends in lower extremity management for ambulatory children with cerebral palsy. Phys Med Rehabil Clin N Am. 2009;20(3):469-491.

62. Papavasiliou AS. Management of motor problems in cerebral palsy: a critical update for the clinician. Eur J Paediatr Neurol. 2009;13(5):387-396.

63. Basaran A, Emre U, Karadavut KI, Balbaloglu O, Bulmus N. Hand splinting for poststroke spasticity: a randomized controlled trial. Top Stroke Rehabil. 2012;19(4): 329-337. 